\title{
ADSORÇÃO DE QUINOLINA EM SÍLICA-ALUMINA A PARTIR DE SOLUÇÕES COM N-HEXADECANO: ESTUDO DA CINÉTICA E DA ISOTERMA POR CALORIMETRIA
}

\author{
L. B. MADUREIRA ${ }^{1}$, R. A. REIS ${ }^{1}$ e M. L. L. PAREDES ${ }^{1}$ \\ ${ }^{1}$ Programa de Pós-graduação em Engenharia Química/UERJ \\ *E-mail para contato: luanabarreto_010@hotmail.com
}

\begin{abstract}
RESUMO - Nos estudos de adsorção, ensaios de cinética e isoterma em banhos agitados são frequentemente utilizados na avaliação do desempenho dos adsorventes. Alternativamente, este trabalho explora uma estratégia envolvendo uma técnica calorimétrica associada a modelagem da transferência de calor e massa visando uma melhor interpretação desses fenômenos e a obtenção de parâmetros de transporte de quinolina solubilizada em n-hexadecano em uma sílica-alumina comercial a $40^{\circ} \mathrm{C}$. A partir das curvas calorimetrias experimentais em conjunto ao modelo proposto, observou-se uma uniformidade energética dos sítios do adsorvente interagindo com a quinolina. Além disso, pelo ajuste do coeficiente de difusão efetivo da quinolina no sólido adsorvente, representou-se satisfatoriamente as curvas calorimétricas experimentais com o modelo proposto. A partir deste trabalho, é possível afirmar que a calorimetria, alinhada a um modelo fenomenológico apropriado, auxilia na interpretação dos fenômenos de transporte envolvidos na adsorção em fase líquida.
\end{abstract}

\section{INTRODUÇÃO}

As leis ambientais referentes à eliminação de compostos nitrogenados e sulfurados na queima de combustíveis fósseis estão cada vez mais rígidas. Grandes agências internacionais, tanto as europeias (CEN - Comitê Europeu de Normalização) quanto às americanas (US EPA - Agência de Proteção Ambiental dos EUA) desenvolveram regulamentações específicas para minimizar os teores dos gases de efeito estufa além dos teores de enxofre dos combustíveis fósseis (Turaga et al., 2003; Hernandez, 2010).

Atualmente, dentre as alternativas para remoção dos contaminantes presentes no diesel, a adsorção apresenta lugar de destaque como etapa de polimento ou pré-tratamento do hidrotratamento. Uma de suas principais vantagens é operar a baixa pressão e a temperatura ambiente (Ke e Xin, 2010). Nos estudos de adsorção, ensaios de cinética e isoterma usando banhos agitados são frequentemente utilizados na avaliação do desempenho dos novos adsorventes. Entretanto, tais ensaios apresentam algumas limitações quando os sistemas envolvem elevadas relações entre a concentração inicial de soluto e massa de adsorvente. Neste caso, as diferenças entre as concentrações iniciais e finais podem ser imperceptíveis frente aos limites de detecção do equipamento analítico. Alternativamente, foi utilizada neste trabalho uma técnica calorimétrica associada a modelagem dos fenômenos de transferência de calor e massa envolvidos no ensaio, visando uma melhor interpretação desses fenômenos e a 
obtenção de parâmetros de transporte. Neste cenário, a calorimetria permitiu não somente medir o calor envolvido durante o processo, como também analisar os mesmos fenômenos estudados nas técnicas com banho agitado. Uma das vantagens da técnica é a utilização de pouco material e reagente, além de envolver menor manipulação da amostra e não apresentar limitação em relação ao ensaio em leito agitado.

\section{EXPERIMENTAL}

O calorímetro utilizado neste trabalho (Micro Reaction Calorimeter da THT) dispõe de uma seringa de $250 \mu \mathrm{L}$ termostatizada para as injeções dos reagentes em um frasco de vidro de $1,5 \mathrm{~mL}$ disposto sobre o sistema de sensores. Neste trabalho, o calorímetro foi operado no módulo de compensação de potência, isto é, o dispositivo, acionado por um sistema de sensores de Peltier, fornece ou retira calor da amostra visando manter sua temperatura fixada em um valor de referência. Um agitador magnético produz na amostra uma agitação controlada de $200 \mathrm{rpm}$ para garantir sua homogeneização. A variação de temperatura da amostra em relação à temperatura de referência causada pelo fenômeno de mistura ou adsorção é o parâmetro de realimentação para que a potência gerada pelo microcalorímetro restaure o equilíbrio da temperatura no compartimento da amostra. Desta maneira, mede-se a taxa de calor (potência) transmitida entre a amostra e o sistema de controle. O calor gerado corresponde a integral da potência ao longo do tempo, como demonstrado na Equação 1.

$$
Q=\int_{t=0}^{t=T} P . \mathrm{d} t
$$

sendo $Q$ representa a energia elétrica em $\mathrm{mJ}$ e $P$ a potência elétrica em $\mathrm{mW}$.

Inicialmente, foram realizados ensaios de entalpia em excesso à $25^{\circ} \mathrm{C}$ para misturas de tetrahidrofurano (THF, 99\% de pureza, VETEC) com n-hexano (95\% de pureza, VETEC) e acetato de etila (99,5\% de pureza, VETEC) com n-hexano, com o objetivo de validação da metodologia experimental e obtenção dos parâmetros intrínsecos do equipamento presentes no modelo de transferência de calor do sistema calorimétrico adotado. Os dados de entalpia em excesso para esses sistemas foram comparados com aqueles obtidos por Castro et al. (1994) e Grolier et al. (1974). Os experimentos foram realizados em triplicata.

\subsection{Adsorção Calorimétrica}

Foram selecionados para a realização dos experimentos de adsorção calorimétrica, a quinolina fornecida pela Sigma-Aldrich com $98 \%$ de pureza e o n-hexadecano fornecido pela VETEC com $99 \%$ de pureza. A quinolina foi utilizada por representar um dos contaminantes nitrogenados típicos do diesel e o n-hexadecano foi utilizado como solvente no preparo das soluções por ser um dos principais constituintes desta fração de petróleo. Também foi utilizado uma sílica-alumina fornecida pela SASOL, denominada Siral-40. A sílica-alumina passa por um processo de pré-tratamento visando sua alteração estrutural e, posteriormente, é peneirada na faixa granulométrica de 100 mesh - 325 mesh. Finalmente, a amostra é seca para o ensaio calorimétrico (Santos et al., 2012). Após seu resfriamento, o adsorvente é pesado em balança analítica com precisão de $0,0001 \mathrm{~g}$ dentro do frasco de vidro. Visando garantir a completa remoção do ar presente nos poros do adsorvente, o sólido pesado é previamente 
misturado com um volume pré-determinado de n-hexadecano puro (Santos et al., 2012). Este procedimento permite também eliminar a interferência produzida pelas interações entre o sólido e o solvente (n-hexadecano) em relação as medidas de calor de adsorção da quinolina em Siral-40. O volume utilizado deve ser suficiente para molhar completamente o adsorvente. Depois desta etapa, o calorímetro é programado para realizar a injeção do volume de solução de quinolina através da seringa microvolumétrica de $250 \mu \mathrm{L}$. Todos os experimentos de adsorção calorimétrica foram realizados a $40{ }^{\circ} \mathrm{C}$ e foram injetados $70 \mu \mathrm{L}$ de solução de quinolina em diferentes concentrações. As soluções de quinolina foram preparadas de modo a garantir concentrações iniciais pré-estabelecidas $\left(C_{0}\right)$ levando em conta o volume de nhexadecano utilizado na etapa de molhamento. As concentrações iniciais foram estabelecidas através da Equação 2 utilizando os dados experimentais de isoterma de adsorção $(q \times C)$ obtidos previamente para esse sistema em leito agitado.

$$
q=\frac{M_{L}\left(C_{0}-C\right)}{M_{a d s}}
$$

sendo $q$ é a razão entre a massa adsorvida do nitrogênio $(\mathrm{N})$ por massa de sólido adsorvente $(\mathrm{mg} / \mathrm{g})$ e $C$ a concentração de $\mathrm{N}$ da fase líquida no equilíbrio termodinâmico em $\mathrm{mg} / \mathrm{kg}, M_{L} \mathrm{e}$ $M_{a d s}$ são as massas de fase líquida em $\mathrm{kg}$ e de sólido adsorvente em g utilizada em cada ensaio calorimétrico, respectivamente, e $C_{0}$ é a concentração inicial da fase líquida de $\mathrm{N} \mathrm{em} \mathrm{mg/kg}$.

\subsection{Modelagem das Curvas da Curvas Calorimétricas}

No calorímetro, um frasco contendo líquido (ou líquido e sólido) recebe a injeção de outro líquido, inicialmente, na mesma temperatura. A mistura (com ou sem a presença do sólido) acarreta um fenômeno térmico devido a formação de novas interações intermoleculares. O calorímetro preparado para ceder ou retirar calor do sistema, compensa a potência gerada durante o fenômeno de mistura e/ou adsorção de modo a manter a temperatura do sistema constante, resultando na curva de potência térmica versus tempo. A representação mais simples desse processo requer que seja realizado o balanço de energia na solução (com ou sem sólido) dentro do frasco, sem entrada ou saída de fluxos mássicos, perdendo calor por condução para o vidro que, por sua vez transfere para a placa metálica e, finalmente, ao sensor (resistências em série para a transferência de calor). Devido à presença de um agitador magnético, é razoável considerar a solução (com ou sem sólido) como um tanque de mistura perfeita com propriedades homogêneas em todo o volume. Considerando que o fluido é aproximadamente incompressível e possua propriedades termofísicas constantes, o balanço de energia pode ser expresso pela Equação 3:

$\rho \iiint_{V} C_{p} \frac{\partial T}{\partial t} d V=\rho C_{p} \frac{\partial T}{\partial t} \Delta V=C_{p, s o l}^{t} \frac{\partial T}{\partial t}=\dot{Q}+\dot{E}_{g e r}$

sendo que o sobrescrito $t$ indica extensivo e o subscrito sol indica solução (com ou sem sólido), $\dot{Q}$ corresponde ao calor que é transferido para o frasco de vidro por condução, $\dot{E}_{g e r}$ corresponde ao termo de geração e consumo, (positivo para geração e negativo para consumo), $t$ corresponde ao tempo, $T$ a temperatura, $C_{p, s o l}^{t}$ é a capacidade calorífica da 
solução, $\rho$ é a massa específica e $V$ é o volume de controle que envolve a transferência de calor. Neste trabalho, consideram-se dois volumes de controle em série: o fundo do fraco de vidro e uma placa de alumínio que separa o frasco do sensor de temperatura.

Desprezando o calor transferido pelas laterais do frasco e pela superfície da solução, e desprezando a variação de temperatura no vidro nas suas laterais, tem-se que o calor transferido entre a solução e o frasco ocorrendo apenas pelo fundo do frasco de espessura $L_{V}$, ou seja, a transferência de calor é unidirecional na coordenada $z$. O fundo do frasco, por sua vez, troca calor com a placa de alumínio de espessura $L_{M}$ sobre a qual repousa. Assim, neste sistema, o balanço de energia em cada porção de seu volume é dado pela Equação 4, sendo $C_{p}$ considerando constante e $k$ corresponde à condutividade térmica do frasco de vidro ou da placa de alumínio.

$\rho C_{p} \frac{\partial T}{\partial t}=k \frac{\partial^{2} T}{\partial z^{2}}$

O primeiro caso de titulação calorimétrica que é tratado neste trabalho é a titulação entre dois líquidos, sendo um problema de determinação de entalpia em excesso. Neste caso, ocorre uma variação de temperatura instantânea em relação à temperatura de referência devido ao calor de mistura dos líquidos transferido instantaneamente no instante inicial. A resolução do sistema de equações que descreve de forma simplificada esse problema de transferência de calor será obtido pela discretização da Equação 4 no espaço por volumes finitos e no tempo por Crank- Nicolson (Chapra; Canale, 2008) quando aplicada às duas resistências em série $\left(L_{V}\right.$ e $L_{M}$ ) sob as seguintes condições iniciais e de contorno: (i) a temperatura da face do fundo do frasco em contato com a solução é sempre igual à temperatura da solução em qualquer instante; (ii) no instante inicial, todos os outros elementos de volume nos sólidos encontramse na temperatura de referência (setpoint); e (iii) a temperatura da face externa da placa metálica, em contato com o Peltier, é sempre a temperatura de referência para qualquer instante.

A partir destas premissas foi possível propor um modelo para o fenômeno de transferência de calor que ocorre no processo de entalpia em excesso, quando não há o termo de geração de calor ( $\dot{E}_{g e r}=0$ ), e desta forma foi possível descrever o perfil de temperatura ao longo do eixo " $z$ " do processo descrito. O sistema de equações foram resolvidas no Scilab e o parâmetro $L_{M}$ foi estimado através da minimização da soma dos desvios em $\dot{Q}$ (em mW), para cada fração molar estudada das diferentes misturas. O método utilizado na minimização da função objetivo foi o Simplex de Nelder-Mead (Nelder e Mead, 1965).

No experimento de adsorção calorimétrica, o termo $\dot{E}_{g e r}$ corresponde ao calor transferido durante o processo de adsorção, $\dot{Q}_{a d s}$, definido pela seguinte equação $\dot{Q}_{a d s}=\partial \bar{q} / \partial t\left(M_{a d s} H a d s\right)$. O termo $\partial \bar{q} / \partial t$ representa a variação da razão média na partícula da massa de soluto adsorvida por massa de sólido adsorvente $(q)$ ao longo do tempo, $M_{a d s}$ representa a quantidade de sólido adsorvente (g) e Hads representa o calor liberado por quantidade de soluto adsorvido $(\mathrm{J} / \mathrm{mg})$. O termo $\partial \bar{q} / \partial t$ foi obtido a partir do balanço de 
massa do adsorbato na fase adsorvida. O processo de liberação do calor de adsorção foi considerado sendo governado pela transferência de massa do adsorbato da região volumar da fase fluida até um sítio localizado na superfície da partícula. Apesar da distribuição de diâmetros de poros das partículas apresentar elevada dispersão (Santos et al., 2012), considerou-se, para efeitos de transferência de massa, que o volume da partícula adsorvente fosse representado por um meio pseudo-homogêneo. A hipótese inclui também a consideração de que os sítios de adsorção estão uniformemente distribuídos ao longo do volume da partícula e com mesma energia de interação pelo adsorbato. A resistência a transferência de massa externa a partícula foi negligenciada (regime turbulento de agitação no frasco), considerou-se que as partículas são de mesmo tamanho (diâmetro médio da faixa granulométrica utilizada) e se encontram em um recipiente em mistura perfeita. Sob estas hipóteses, foi proposto um balanço de massa do adsorbato na fase adsorvida utilizando coordenadas esféricas com origem no centro da partícula. Neste trabalho, considerou-se a resistência a transferência de calor no interior de cada partícula e entre partículas negligenciável. A partir destas premissas, propõem-se a seguinte equação para o balanço de massa de quinolina na fase adsorvida:

$$
\frac{\partial q}{\partial t}=\frac{1}{r} \frac{\partial}{\partial r}\left(r^{2} D c \frac{\partial q}{\partial r}\right)
$$

sendo $D c$ a difusividade efetiva do adsorbato na partícula (fase pseudo-homogênea) e $q(r, t)$ a razão mássica de adsorbato na fase adsorvida. Em todos os ensaios desenvolvidos neste trabalho, partiu-se do sólido inicialmente isento de adsorbato, isto é, $q(r, 0)=q_{0}=0$. A relação da massa de adsorbato adsorvido por massa de adsorvente na superfície da partícula $\left(r=r_{c}\right)$ em um determinado instante de tempo $\left(q^{*}\right)$, é aquela que encontra-se em equilíbrio termodinâmico com a concentração da fase líquida externa a partícula, isto é $q\left(r_{c}, t\right)=q^{*}$. Na integração da Equação 5, $q^{*}$ foi considerado constante ao longo do tempo (Ruthven, 1984). No centro na partícula, em qualquer instante de tempo, podemos adotar a condição de contorno de simetria, $(\partial q / \partial r)_{r=0}=0$. A solução do perfil transiente da concentração de adsorbato por massa de adsorvente é representado pela Equação 6 (Ruthven, 1984).

$$
\theta=\frac{q-q^{*}}{q_{0}-q^{*}}=\frac{2 r_{c}}{\pi} \sum_{n=1}^{\infty} \frac{(-1)^{n+1}}{n r} \operatorname{sen}\left(\frac{n \pi r}{r_{c}}\right) \exp \left[-\left(\frac{n \pi}{r_{c}}\right)^{2} D c t\right]
$$

O valor médio na partícula da massa de soluto adsorvida pela quantidade de sólido adsorvente, $\bar{q}$, é obtido pelo teorema do valor médio. $\mathrm{O}$ termo $\partial \bar{q} / \partial t$ foi então obtido pela seguinte expressão:

$$
\frac{\partial \bar{q}}{\partial t}=\left\{\frac{q^{*}}{\left\{1-\frac{\partial q^{*}}{\partial \bar{q}}\left[1-\frac{6}{\pi^{2}} \sum_{n=1}^{\infty} \frac{1}{n^{2}} \exp \left(-\frac{n^{2} \pi^{2} D_{c} t}{r_{c}^{2}}\right)\right]\right\}}\right\} \cdot \frac{6 D_{c}}{r_{c}^{2}} \sum_{n=1}^{\infty} \exp \left(-\frac{n^{2} \pi^{2} D_{c} t}{r_{c}^{2}}\right)
$$


A partir do ajuste do modelo de Langmuir-Freundlich dos dados experimentais obtidos em leito agitado para a isoterma de adsorção de quinolina em SIRAL-40 dispersos em nhexadecano a $40^{\circ} \mathrm{C}$, foi possível estimar os parâmetros da referida isoterma que foi utilizada na relação entre $q^{*}$ e $C(t)$. Finalmente, a Equação 2 completa a relação entre $q^{*} \mathrm{e}$ $\bar{q}(t)$ necessária o cálculo de $\partial \bar{q} / \partial t$ pela Equação (7).

\section{RESULTADOS E DISCUSSÕES}

\subsection{Entalpia em Excesso de Misturas Líquidas Binárias}

A partir da comparação dos dados gerados neste trabalho e aqueles disponíveis na literatura (Castro et al., 1994; Grolier et al., 1974), pode-se afirmar que a metodologia adotada permitiu não somente obter dados de entalpia molar em excesso com baixos valores de incerteza padrão, assim como reproduzir os dados da literatura (Tabela 1).

Tabela 1 - Entalpia Molar em Excesso $\left(H^{E}\right)$ em função da composição molar a $25^{\circ} \mathrm{C}$. A incerteza foi calculada como 1,96 vezes o desvio padrão.

\begin{tabular}{ccccc}
\hline \multirow{2}{*}{ Fração Molar $\left(x_{1}\right)$} & Média & $\begin{array}{c}H^{E}(\mathrm{~J} / \mathrm{mol}) \\
\text { Incerteza Padrão }\end{array}$ & Literatura & Referência \\
\hline \multicolumn{5}{c}{ THF (1)/n-Hexano (2) } \\
\hline 0,9382 & 189 & 11 & 188 & Castro et al. $(1994)$ \\
0,8997 & 315 & 52 & 291 & Castro et al. $(1994)$ \\
0,8590 & 415 & 34 & 390 & Castro et al. (1994) \\
\hline 0,9061 & 476 & 20 & 452 & Grolier et al. $(1974)$ \\
0,8660 & 677 & 93 & 609 & Grolier et al. $(1974)$ \\
0,8282 & 811 & 27 & 740 & Grolier et al. $(1974)$ \\
\hline
\end{tabular}

Cada curva experimental de potência de compensação em função do tempo para misturas líquidas binárias foi satisfatoriamente ajustada ao modelo proposto (item 2.2), estimando-se a espessura da placa metálica $\left(L_{M}\right)$. Desta forma, foram obtidos 18 diferentes valores para $L_{M}$ (diferentes composições das misturas e suas réplicas), com média centrada em $0,119 \mathrm{~m}$ e desvio padrão de $0,004 \mathrm{~m}$. O baixo desvio relativo encontrado $(3,4 \%)$ é considerado satisfatório, uma vez que $L_{M}$ representa uma variável estrutural do equipamento, entretanto o valor de aproximadamente $0,12 \mathrm{~m}$ encontra-se bem acima do esperado, evidenciando possíveis limitações do modelo de transferência de calor adotado.

\subsection{Obtenção Experimental da Curvas de Cinéticas por Calorimetria}

A partir das curvas calorimétricas experimentais obtidas durante o processo de adsorção associadas ao modelo matemático de transferência de calor e massa adotado (Equações 2-7), foi possível determinar as concentrações de equilíbrio $C$ e $\bar{q}$ para cada condição experimental, assim como ajustar os respectivos valores para o coeficiente de difusão efetivo da quinolina na SIRAL 40. De posse dessas informações, calculou-se a quantidade de calor liberada por miligrama de nitrogênio adsorvido ( $H a d s$ ), sendo este calculado pela equação $H a d s=Q / \bar{q} M_{a d s}$. A Figura 1 representa a quantidade de calor liberada por miligrama de 
soluto adsorvido para cada concentração de equilíbrio.

Na Figura 1, observa-se que a maioria dos dados encontram-se flutuando pouco em torno de um valor médio de $0,48 \mathrm{~J} / \mathrm{mg}$ de $\mathrm{N}$. Assim, pode-se aferir que os sítios do adsorvente são energicamente similares, uma vez que, tanto em concentrações de equilíbrio mais elevadas, quanto em concentrações mais baixas, os valores de Hads são próximos. Os pontos vazados foram desconsiderados devido ao elevado ruído dos dados do termograma em relação a linha base.

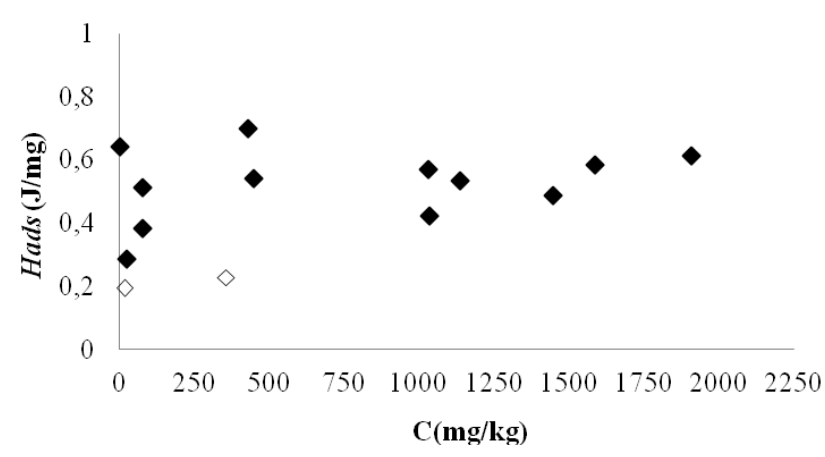

Figura 1 - Calor liberado por quantidade de soluto adsorvido ( Hads ) em função da concentração de equilíbrio, $\diamond$ denota os pontos que apresentaram grande flutuação no termograma.

Para cada termograma obtido em diferentes valores de $C_{0}$, estimou-se um valor do coeficiente de difusão efetivo $(D c)$ da quinolina na SIRAL 40, utilizando a espessura média do metal $\left(L_{M}=0,119 \mathrm{~m}\right)$. A Figura 2 representa os valores do coeficiente de difusão em relação a concentração de equilíbrio. Os valores ajustados encontram-se flutuando em torno de um coeficiente de difusão efetivo médio de $4,10.10^{-13} \mathrm{~m} / \mathrm{s}^{2}$. Dois valores $\left(5,20.10^{-12} \mathrm{~m} \cdot \mathrm{s}^{-2} \mathrm{e}\right.$ $1,70.10^{-15} \mathrm{~m} \cdot \mathrm{s}^{-2}$ ) apresentaram flutuações mais pronunciadas em relação à média.

De modo geral, o modelo proposto, quando ajustado as curvas experimentais de potência térmica gerada ao longo da cinética de adsorção à $40^{\circ} \mathrm{C}$, representou satisfatoriamente o comportamento experimental, mas os desvios encontrados sugerem que outros efeitos necessitam ser incorporados nos modelos de transferência de calor e massa.

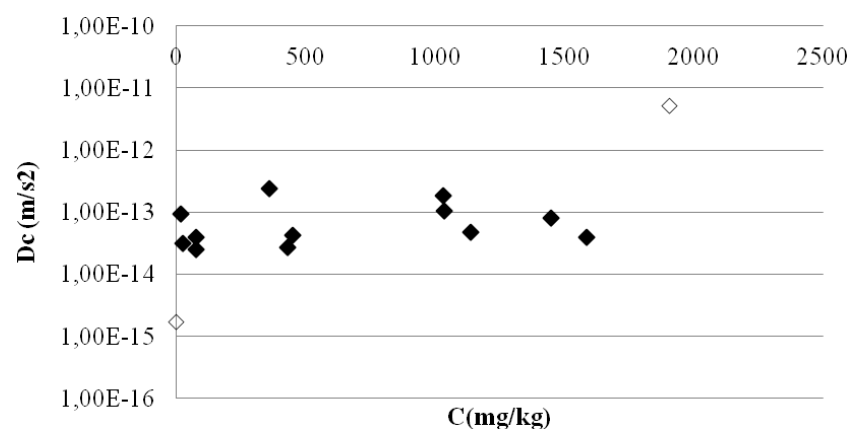

Figura 2 - Coeficientes de difusão $(D c)$ em função da concentração de equilíbrio, $\diamond$ representa os pontos com maior desvio. 


\section{CONCLUSÃO}

Neste trabalho, ensaios calorimétricos foram desenvolvidos visando auxiliar o entendimento dos fenômenos de transferência de calor e massa envolvidos no processo de adsorção de quinolina em sílica-alumina comercial a partir de soluções de n-hexadecano. Primeiramente, a metodologia foi utilizada para reproduzir com sucesso dados da literatura de entalpia em excesso das misturas binárias de THF e n-hexano e, acetato de etila e n-hexano. Nesta etapa, foi possível também obter um único valor ajustado para a espessura da placa metálica. Entretanto, seu valor encontra-se ainda acima do esperado.

A partir das curvas calorimetrias experimentais e do cálculo das concentrações de equilíbrio na fase líquida para cada condição avaliada, foi possível observar que, na maior parte dos experimentos, o Hads é aproximadamente constante, o que sugere uniformidade energética dos sítios do adsorvente. Além disso, pelo ajuste do coeficiente de difusão efetivo da quinolina no sólido adsorvente, foi possível representar satisfatoriamente as curvas experimentais de potência térmica gerada ao longo da cinética de adsorção à $40^{\circ} \mathrm{C}$. A partir deste trabalho, é possível afirmar que a calorimetria alinhada a um modelo fenomenológico mais completo representa uma importante ferramenta na interpretação do fenômenos de transporte envolvidos no processo de adsorção em fase líquida.

\section{REFERÊNCIAS}

CASTRO, I.; PINTOS, M.; AMIGO, A.; BRAVO, R.; ANDRADE, M.I. Excess enthalpies of (tetrahydrofuran or tetrahydropyran + n-alkane) at the temperature $298,15 \mathrm{~K}$. J. Chem. Therm., v. 26, p. 29-33, 1994.

CHAPRA, S.C.; CANALE, R.P. Métodos numéricos para engenharia. São Paulo: $5^{\circ}$ Ed, Mac Graw Hill Interamericana do Brasil LTDA, 2008.

GROLIER, J.P.E.; BALLET, D.; VIALLARD, A. Thermodynamics of ester-containing mixtures. Excess enthalpies and excess volumes for alkyl acetates and alkyl benzoates + alkanes, + benzene,+ toluene, and + ethylbenzene. J. Chem. Therm, v. 6, p. 895-908, 1974.

HERNANDEZ, S.P.; FINO, D.; RUSSO, N. High performance sorbents for diesel oil desulfurization. Chem. Eng. Sci., v. 65, p. 603-609, 2010.

KE, T.; XIN, H. Deep desulfurization of model gasoline by adsorption on mesoporous CeMCM-41. Petrol. Sci. Tech., v. 28, p. 573-581, 2010.

NELDER, J.A.; MEAD, R. A simplex method for function minimization. Comput. J., v.7, p. 308-313, 1965.

RUTHVEN, D.M. Principles of adsorption and adsorption process. John Whiley \& Sons, USA, 1984.

SANTOS, A.L.; REIS, R.A.; ROSSA, V.; REIS, M.M.; COSTA, A.L.H.; VELOSO, C.O., HENRIQUES, C.A.; ZOTIN, F.M.Z.; PAREDES, M.L.L.; SILVEIRA, E.B.; CHIARO, S.S.X. Silica-alumina impregnated with cerium, nickel, and molybdenum oxides for adsorption of sulfur and nitrogen compounds from diesel. Mater. Lett., v. 83, p. 158160, 2012.

TURAGA, U.T.; MA, X.; SONG, C. Influence of nitrogen compounds on deep hydrodesulfurization of 4,6-dimethyldibenzothiophene over Al2O3- and MCM-41 supported Co-Mo sulfide catalysts. Catal. T., v. 86, p. 265-275, 2003. 